\title{
A non-ideally excited pendulum controlled by SDRE technique
}

\author{
Angelo Marcelo Tusset ${ }^{1} \cdot$ Átila Madureira Bueno ${ }^{2}$ João Paulo Martins dos Santos ${ }^{3}$. \\ Masayoshi Tsuchida $^{4} \cdot$ José Manoel Balthazar $^{5}$
}

Received: 24 June 2014 / Accepted: 24 February 2016 / Published online: 22 March 2016

(C) The Brazilian Society of Mechanical Sciences and Engineering 2016

\begin{abstract}
In this work, the perturbation theory is applied to the analysis of an electromechanical pendulum system. The frequency response behavior of the system is studied, and the existence of unstable poles is detected using the Routh-Hurwitz criterion. Numerical simulations show the existence of nonlinear behaviors such as hysteresis and the Sommerfeld effect in the resonance region. To damp the electromechanical system oscillations due to the nonlinear characteristics of the system the State Dependent Riccati Equation (SDRE) technique is used. The SDRE control strategy is applied considering two control signals, a feedback control that force the state trajectory of the system to a previously defined periodic orbit, and a nonlinear feedforward control that keeps the system motion synchronized to the periodic orbit. Additionally, the robustness of the control technique is tested for parametric uncertainties.
\end{abstract}

Keywords Nonlinear control · Non-ideal systems . SDRE $\cdot$ Hysteresis $\cdot$ Sommerfeld effect

Technical Editor: Aline Souza de Paula.

Átila Madureira Bueno

atila@sorocaba.unesp.br

Angelo Marcelo Tusset

tusset@utfpr.edu.br

João Paulo Martins dos Santos

jp2@usp.br

Masayoshi Tsuchida

tsuchida@ibilce.unesp.br

José Manoel Balthazar

Josemanoel.balthazar@feb.unesp.br

1 Departamento Acadêmico de Matemática - DAMAT,

Universidade Tecnológica Federal do Paraná - UTFPR,

Ponta Grossa, PR, Brazil

\section{Introduction}

Usually, the mathematical models of dynamical systems suppose that the excitation signals ${ }^{1}$ are ideal, that is, the power source is not affected by the dynamical system behavior. For instance, the frequency of an oscillatory excitation signal is determined by the power source, not depending on the motion of the dynamical system. This kind of system is said to have an ideal (unlimited) power supply.

In the recent decades the interest on non-ideal systems has grown. In non-ideal systems the excitations signals have limited power supply, and therefore, the behavior of the excitation signal is affected by the motion of the dynamical system. In 1902 the first non-ideal problem was reported when Sommerfeld observed the interactions between a motor and its elastic foundation (a cantilever beam). The system exhibited unstable motions in the resonance region, usually known as jump phenomenon or Sommerfeld effect [1].

2 Instituto de Ciência e Tecnologia de Sorocaba - ICTS, Universidade Estadual Paulista - UNESP, Sorocaba, SP, Brazil

3 Academia da Força Aérea (AFA), Pirassununga, SP, Brazil

4 Departamento de Ciência da Computação e Estatística - DCCE, Universidade Estadual Paulista - UNESP, São José do Rio Preto, SP, Brazil

5 Divisão de Engenharia Aeronáutica, Instituto Tecnológico de Aeronática - ITA, São José dos Campos, SP, Brazil

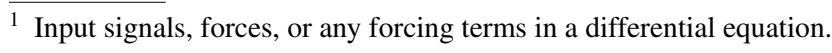


According to [3], dynamical systems with non-ideal power sources may develop a number of different behaviors, such as Sommerfeld effect, hysteresis and chaos. The influence on the shape of the response curve when a control parameter is modified is called hysteresis, and the characteristics of the frequency response are sufficient to its characterization [2]. In addition, the Sommerfeld effect can be detected observing the amplitude of the oscillations as a function of the control parameters values [4].

In [5], the observed Sommerfeld effect of a cantilever beam coupled to a non-ideal unbalanced motor is analysed considering numerical and experimental tests. The sensitivity of the system to its parameters variations is determined by numerical simulations.

In [6], the dynamics of a nonlinear oscillator excited by a non-ideal source is studied both numerically and analytically. The Sommerfeld effect was detected by analyzing the amplitude of motion as a function of the excitation frequency. An approach for the parameters determination of the non-ideal system for which the Sommerfeld effect does not exist is developed.

In [7], a feedback control is used to generate chaotic oscillations in a simple electromechanical device, and numerical simulations detected the existence of Sommerfeld effect.

In [8], the Bogoliubov Averaging Method is applied to study the vibrations in an elastic foundation forced by a non-ideal energy source. Considering the equations of motion, numerical simulations, and the averaged equations, the authors conclude that the Bogoliubov averaging method is an excellent tool to study the characteristics of motion of a non-ideal system.

In [9], the nonlinear control method based on the saturation phenomenon is applied to a non-ideal portal frame system. The equations of motion are analyzed using the method of averaging and numerical simulation. The results show that the motion of the portal frame becomes saturated, and most of the energy supplied to the portal frame by the non-ideal source is transferred to the controller.

In [10], a magnetically levitated body excited by a non-ideal energy source is numerically analyzed, and it is observed that the maximum vibration amplitude occurred in the regions where the jump phenomenon occurs. To decrease the amplitude of oscillations on the resonances regions, passive and active controllers are proposed.

In [11], the attenuation and suppression of the Sommerfeld effect in a non-ideal vibrating system using a magnetorheological damper (MRD) is studied. Numerical simulations exhibited different variations of the Sommerfeld effect for different current values applied to the MRD.

In [12], the dynamic behavior and vibration control of an electromechanical system under non-ideal excitation is studied. The Routh-Hurwitz stability criterion is used to determine the stability of the controlled system.

In this work the motion control of a non-ideally excited pendulum is proposed. Two control strategies are designed, a feedback controller that captures the system motion to a previously defined orbit [13], and a feedforward controller that keeps the system motion synchronized to that orbit. The feedback controller is designed using the StateDependent Riccati Equation (SDRE) technique.

The SDRE was first proposed by [14], and studied by [15-17], and has become popular over the control community during the last decade. The state transitions in the SDRE method depend on a matrix valued state dependent function. Additionally, the nonlinear terms are parametrized in the state vector. It is an effective algorithm for synthesizing feedback control, considering a nonlinear state space equation, to generate successive optimal solutions for the non-ideal system, being successfully applied to nonlinear systems $[13,18$, 19], electromechanical pendulum [20, 21], robotic systems [22-24], micro electro-mechanical systems (MEMS) [25, 26] and atomic force microscopy (AFM) systems [27-30].

\section{Mathematical model, derivation of governing equations and approximate analytical solution}

The dynamics of an electro-mechanical pendulum with non-ideal power source, shown in Fig. 1, is investigated. The system consists of a mass block $m_{1}(\mathrm{~kg})$ that oscillates horizontally because of the rotation of the mass $m_{2}(\mathrm{~kg})$ in a distance $R(\mathrm{~m})$ from the DC motor axis. The mass block $m_{1}$ is connected to the wall by a spring and a damper, with coefficients $k_{1}(\mathrm{~N} / \mathrm{m})$ and $c_{1}(\mathrm{Nm} / \mathrm{s})$, respectively. A pendulum of mass $m_{3}(\mathrm{~kg})$ and length $l(\mathrm{~m})$ is joined to the block.

Defining the generalized coordinates by $\left(\begin{array}{lll}q_{1} & q_{2} & q_{3}\end{array}\right)$, where $q_{1}$ is the horizontal displacement of mass $m_{1}, q_{2}$ is the angular displacement of the mass $m_{2}$, and $q_{3}$ is the angular displacement of the pendulum with mass $m_{3}$. The kinetic energy $T$, the potential energy $V$, and the external forces $N_{i}, i=1,2,3$, are given by:

$$
\begin{aligned}
T= & \frac{1}{2}\left\{m_{1} \dot{q}_{1}^{2}-2 m_{2} R \dot{q}_{1} \dot{q}_{2} \sin q_{2}+\left(m_{2} R^{2}+J_{2}\right) \dot{q}_{2}^{2}\right. \\
& \left.+2 m_{3} l \dot{q}_{1} \dot{q}_{3} \cos q_{3}+m_{3} l^{2} \dot{q}_{3}^{2}\right\} \\
V= & \frac{1}{2} k_{1} q_{1}^{2}+g\left\{m_{2} R \sin q_{2}+m_{3} l\left(1-\cos q_{3}\right)\right\} \\
N_{1}= & -c_{1} \dot{q}_{1}, \quad N_{2}=M\left(\dot{q}_{2}\right)=a-b \dot{q}_{2}, \quad N_{3}=-c_{3} \dot{q}_{3}
\end{aligned}
$$

Applying the Lagrange formulation, the dynamical electro-mechanic pendulum system equations of motion are given by: 


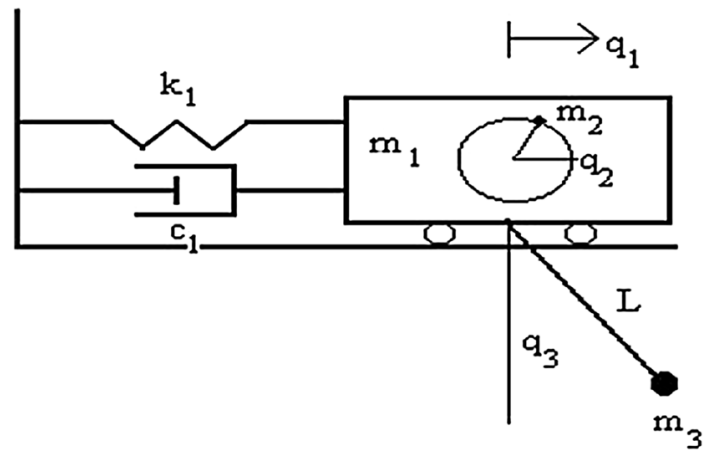

Fig. 1 Electro-mechanical pendulum system

$$
\begin{aligned}
& m_{1} \ddot{q}_{1}-m_{2} R\left(\ddot{q}_{2} \sin q_{2}+\dot{q}_{2}^{2} \cos q_{2}\right) \\
& +m_{3} l\left(\ddot{q}_{3} \cos q_{3}-\dot{q}_{3}^{2} \sin q_{3}\right)+k_{1} q_{1}=-c_{1} \dot{q}_{1} \\
& I \ddot{q}_{2}-m_{2} R \dot{q}_{1} \sin q_{2}+g m_{2} R \cos q_{2}=M\left(\dot{q}_{2}\right) \\
& m_{3} l^{2} \ddot{q}_{3}+m_{3} l \ddot{q}_{1} \cos q_{3}+g m_{3} l \sin q_{3}=-c_{3} \dot{q}_{3}
\end{aligned}
$$

given that $I=m_{2} R^{2}+J_{2}$, where $J_{2}$ is the rotor moment of inertia.

The function $M\left(\dot{q}_{2}\right)$ is the driving torque of the motor, and according to [31], the inductance value is much smaller than the response time constant of the system, and therefore, in the steady state it can be considered that $M\left(\dot{q}_{2}\right)=a-b \dot{q}_{2}$, where $a$ is related to the voltage applied across the armature of the DC motor, and $b$ is constant for the considered DC motor.

The friction force on the mass $m_{1}$ is given by $N_{1}=-c_{1} \dot{q}_{1}$, and the friction on the pendulum is given by $N_{3}=-c_{3} \dot{q}_{3}$, and $g$ is the acceleration due to gravity.

In the following, the method of variation of parameters is applied to obtain an approximate solution of the system. To that, the new dimensionless variables are defined by: $\tau=\omega t, u=\frac{q_{1}}{l}, \varphi=\frac{q_{2}}{Q_{2}}$ and $\theta=\frac{q_{3}}{Q_{3}}$ with $\omega^{2}=\frac{k_{1}}{m_{1}}$, where $l[m], Q_{2}=1[\mathrm{rad}]$ and $Q_{3}=1[\mathrm{rad}]$ are constants, and the dimensionless equations of motion are given by:

$$
\begin{aligned}
u^{\prime \prime}= & -\varepsilon \mu_{1} u^{\prime}-u+\varepsilon p_{2} \varphi^{\prime \prime} \sin (\varphi)+\varepsilon p_{2} \varphi^{\prime 2} \cos (\varphi) \\
& -\varepsilon p_{5} \theta^{\prime 2} \theta+\varepsilon p_{5} \theta^{\prime \prime} \\
\theta^{\prime \prime}= & -\varepsilon \mu_{3} \theta^{\prime}-\omega_{3}^{2} \theta-u^{\prime \prime} \\
\varphi^{\prime \prime}= & \varepsilon \alpha-\varepsilon \beta \varphi^{\prime}+\varepsilon p_{3} u^{\prime \prime} \sin (\varphi)-\frac{\varepsilon p_{4} g}{\omega^{2}} \cos (\varphi)
\end{aligned}
$$

given that $m=m_{1}+m_{2}+m_{3}, \quad \frac{c_{1}}{m \omega}=\varepsilon \mu_{1}, \quad \omega_{3}^{2}=\frac{g}{l \omega^{2}}$, $\frac{m_{2} R}{m l}=\varepsilon p_{2}, \quad \frac{m_{2} R l}{I}=\varepsilon p_{3}, \quad \frac{m_{2} R}{I}=\varepsilon p_{4}, \quad \frac{a}{\omega^{2}}=\varepsilon \alpha, \quad \frac{b}{\omega}=\varepsilon \beta$, $\frac{c_{1}}{m \omega}=\varepsilon \mu_{1}, \quad \frac{c_{3}}{m_{3} l^{2} \omega}=\varepsilon \mu_{3}, \quad \frac{m_{3}}{m}=\varepsilon p_{5}, \quad \omega_{1}^{2}=\frac{k_{1}}{m_{1}}$, $\omega_{1}-\Omega=\varepsilon \sigma_{\Omega}, \quad \frac{M\left(\dot{q}_{2}\right)}{\omega^{2} I}=\varepsilon M_{1}\left(\varphi^{\prime}\right), \quad M_{1}(\dot{\varphi})=\alpha-\beta \varphi^{\prime}$, where $0<\varepsilon<<1$ is an arbitrary small parameter. Additionally, for small displacements $\sin (\theta)=\theta$ and $\cos (\theta)=1$. The dimensionless time $\tau$ derivative is denoted by $\frac{\mathrm{d} y}{\mathrm{~d} \tau}=y^{\prime}$, and $\sigma_{\Omega}$ is a detuning parameter [23].

For $\varepsilon \approx 0$ results that:

$u^{\prime \prime}=-u$

$\theta^{\prime \prime}=-\omega_{3}^{2} \theta-u^{\prime \prime}$

$\varphi^{\prime \prime}=0$

Solving Eq. (4) it can be noted that the motor rotates with constant angular frequency $\varphi^{\prime}=\Omega$. The mass block moves harmonically with frequency $\omega_{1}=1$. The pendulum describes a forced movement, and the force is periodically excited with frequency $\omega_{1}$, resulting that:

$u=a_{1} \cos \left(\omega_{1} \tau+\beta_{1}\right)$

$u^{\prime}=-a_{1} \omega_{1} \sin \left(\omega_{1} \tau+\beta_{1}\right)$

$\theta=a_{3} \cos \left(\omega_{3} \tau+\beta_{3}\right)+a_{1} \delta \cos \left(\omega_{1} \tau+\beta_{1}\right)$

$\theta^{\prime}=-a_{3} \omega_{3} \sin \left(\omega_{3} \tau+\beta_{3}\right)-a_{1} \omega_{1} \delta \sin \left(\omega_{1} \tau+\beta_{1}\right)$

$\varphi=\Omega \tau$

$\varphi^{\prime}=\Omega$

where $\delta=\frac{1}{\omega_{3}^{2}-1}$.

For $\varepsilon \neq 0$, one can expect the oscillation frequency of the mass block to be approximately harmonic, and the angular frequency $\varphi^{\prime}$ to be approximately constant, i.e., the motion correspond to functions that vary smoothly over time, and, in this case, the block oscillation is governed by the motor speed. Furthermore, in the presence of dissipative terms the oscillation is forced, and since the $\theta$ varies smoothly over time, it depends on the oscillation amplitude and phase of the block movement. Thus an approximate for $(5)[2,6,9,31]$ is given by:

$u=a_{1} \cos \left(\varphi+\beta_{1}\right)$

$\theta=a_{3} \cos \left(\omega_{3} \tau+\beta_{3}\right)+a_{1} \delta \cos \left(\varphi+\beta_{1}\right)$

$u^{\prime}=-a_{1} \Omega \sin \left(\varphi+\beta_{1}\right)$

$\theta^{\prime}=-a_{3} \omega_{3} \sin \left(\omega_{3} \tau+\beta_{3}\right)-a_{1} \Omega \delta \sin \left(\varphi+\beta_{1}\right)$

$\varphi=\Omega \tau$

$\varphi^{\prime}=\Omega$

Considering $\quad a_{1}=a_{1}(\tau), \quad \beta_{1}=\beta_{1}(\tau), \quad a_{3}=a_{3}(\tau)$, $\beta_{3}=\beta_{3}(\tau)$ and $\Omega=\Omega(\tau)$, the set of variables $\left(a_{1}, \beta_{1}, a_{3}\right.$, $\left.\beta_{3}, \Omega\right)$, smoothly varying over $\tau$, represent the essential parameters of the system [1]. The values of the essential variables are determined by the method of averaging [2]. Consequently, considering the derivative of the Eq. (6) with respect to $\tau$, results:

$$
\begin{aligned}
u^{\prime}= & a_{1}^{\prime} \cos \left(\varphi+\beta_{1}\right)-a_{1}\left(\Omega+\beta_{1}^{\prime}\right) \sin \left(\varphi+\beta_{1}\right) \\
\theta^{\prime}= & a_{3}^{\prime} \cos \left(\omega_{3} \tau+\beta_{3}\right)-a_{3}\left(\omega_{3}+\beta_{3}^{\prime}\right) \sin \left(\omega_{3} \tau+\beta_{3}\right) \\
& +a_{1}^{\prime} \delta \cos \left(\varphi+\beta_{1}\right)-a_{1} \delta\left(\Omega+\beta_{1}^{\prime}\right) \sin \left(\varphi+\beta_{1}\right)
\end{aligned}
$$

From Eqs. (5)-(9) we have: 
$a_{1}^{\prime} \cos \left(\varphi+\beta_{1}\right)-a_{1} \beta_{1}^{\prime} \sin \left(\varphi+\beta_{1}\right)$

$-\left(\Omega-\omega_{1}\right) a_{1} \sin \left(\varphi+\beta_{1}\right)=0$

$a_{3}^{\prime} \cos \left(\omega_{3} \tau+\beta_{3}\right)-a_{3} \beta_{3}^{\prime} \sin \left(\omega_{3} \tau+\beta_{3}\right)=0$

The derivative of Eq. (7) with respect to $\tau$ is given by:

$$
\begin{aligned}
u^{\prime \prime}= & -a_{1}^{\prime} \omega_{1} \sin \left(\varphi+\beta_{1}\right)-a_{1} \omega_{1}\left(\Omega+\beta_{1}^{\prime}\right) \cos \left(\varphi+\beta_{1}\right) \\
\theta^{\prime \prime}= & -a_{3}^{\prime} \omega_{3} \sin \left(\omega_{3} \tau+\beta_{3}\right)-a_{3} \omega_{3}\left(\omega_{3}+\beta_{3}^{\prime}\right) \cos \left(\omega_{3} \tau+\beta_{3}\right) \\
& -a_{1}^{\prime} \delta \omega_{1} \sin \left(\varphi+\beta_{1}\right)-a_{1} \delta \omega_{1}\left(\Omega+\beta_{1}^{\prime}\right) \cos \left(\varphi+\beta_{1}\right)
\end{aligned}
$$

Replacing Eqs. (6), (8) and (9) into Eq. (3), and comparing with (11), results:

$$
\begin{aligned}
& -a_{1}^{\prime} \gamma \sin \left(\varphi+\beta_{1}\right)-a_{1} \beta_{1}^{\prime} \gamma_{1} \cos \left(\varphi+\beta_{1}\right) \\
& +\left(\omega_{1}^{2}-\gamma_{1} \Omega\right) a_{1} \cos \left(\varphi+\beta_{1}\right)=\varepsilon[H] \\
& \Omega^{\prime}=\varepsilon\left\{\begin{array}{l}
M_{1}(\Omega)-p_{3} \sin (\varphi)\left[a^{\prime} \omega_{1} \sin \left(\varphi+\beta_{1}\right)\right. \\
\left.+a_{1} \beta_{1}^{\prime} \omega_{1} \cos \left(\varphi+\beta_{1}\right)+a_{1} \omega_{1} \Omega \cos \left(\varphi+\beta_{1}\right)\right] \\
-p_{4} \frac{g}{\omega^{2}} \cos (\varphi)
\end{array}\right\} \\
& -a_{3}^{\prime} \omega_{3} \sin \left(\omega_{3} \tau+\beta_{3}\right)-a_{3} \omega_{3} \beta_{3}^{\prime}-\cos \left(\omega_{3} \tau+\beta_{3}\right)=[J]
\end{aligned}
$$

where $\gamma_{1}=\left(1+\varepsilon p_{5} \delta \omega_{1}^{2}\right), \gamma=\left(1+\varepsilon p_{5}\right)$, and

$$
\begin{aligned}
{[H]=} & {\left[p_{2} \Omega^{\prime} \sin (\varphi)+p_{2} \Omega^{2} \cos (\varphi)\right.} \\
& \left.+\mu_{1} a_{1} \omega_{1} \sin \left(\varphi+\beta_{1}\right)\right]+m_{3}[G] \\
{[G]=} & {\left[a_{3} \omega_{3} \sin \left(\omega_{3} \tau+\beta_{3}\right)+a_{1} \delta \omega_{1} \sin \left(\varphi+\beta_{1}\right)\right]^{2} } \\
& \times\left[a_{3} \cos \left(\omega_{3} \tau+\beta_{3}\right)+a_{1} \delta \cos \left(\varphi+\beta_{1}\right)\right] \\
& +a_{3}^{\prime} \omega_{3} \sin \left(\omega_{3} \tau+\beta_{3}\right)+a_{3} \omega_{3} \beta_{3}^{\prime} \cos \left(\varphi+\beta_{1}\right) \\
& +a_{3} \omega_{3}^{2} \cos \left(\omega_{3} \tau+\beta_{3}\right) \\
{[J]=} & -a_{1} \omega_{1} \sin \left(\varphi+\beta_{1}\right)-a_{1} \beta_{1}^{\prime} \omega_{1} \cos \left(\varphi+\beta_{1}\right) \\
& +\varepsilon \mu_{3}\left[a_{3} \omega_{3} \sin \left(\omega_{3} \tau+\beta_{3}\right)+a_{1} \delta \omega_{1} \sin \left(\varphi+\beta_{1}\right)\right] \\
& +\delta \omega_{1}\left[a_{1}^{\prime} \sin \left(\varphi+\beta_{1}\right)+a_{1} \beta_{1}^{\prime} \cos \left(\varphi+\beta_{1}\right)\right] \\
& +a_{1} \delta\left[\omega_{1} \Omega-\omega_{3}^{2}\right] \cos \left(\varphi+\beta_{1}\right)
\end{aligned}
$$

Considering the following trigonometric identities:

$$
\begin{aligned}
& \sin ^{2}(\theta)=\frac{1}{2}-\frac{1}{2} \cos (2 \theta) \\
& \sin ^{4}(\theta)=\frac{1}{8}(\cos (4 \theta)-4 \cos (2 \theta)+3) \\
& \sin ^{3}(\theta) \cos (\theta)=\frac{1}{8}(2 \sin (2 \theta)-\sin (4 \theta)) \\
& \cos ^{4}(\theta)=\frac{1}{8}(\cos (4 \theta)+4 \cos (2 \theta)+3) \\
& \cos ^{3}(\theta) \sin (\theta)=\frac{1}{8}(2 \sin (2 \theta)+\sin (4 \theta))
\end{aligned}
$$

and solving Eqs. (10) and (12) for $a_{1}^{\prime}, \beta_{1}^{\prime}, \Omega^{\prime}, a_{3}^{\prime}$ and $\beta_{3}^{\prime}$, the modulations of amplitude and phase of the system are given by [2]:

$$
\begin{aligned}
a_{1}^{\prime}= & -\varepsilon \frac{p_{5} \delta \omega_{1}^{2} a_{1}}{2 \gamma_{1}} \sin \left[2\left(\varphi+\beta_{1}\right)\right]-\frac{\varepsilon}{\gamma_{1}} \\
& \times\left[p_{2} \Omega^{2} \cos (\varphi)+\mu_{1} a_{1} \omega_{1} \sin \left(\varphi+\beta_{1}\right)\right] \sin \left(\varphi+\beta_{1}\right) \\
& -\varepsilon \frac{p_{5}}{\gamma_{1}} \sum_{i=1}^{14} G_{i}+O\left(\varepsilon^{2}\right) \\
\beta_{1}^{\prime}= & -\Omega+\frac{\omega_{1}^{2}}{\gamma_{1}}+\frac{\varepsilon p_{5} \delta \omega_{1}^{2}}{2 \gamma_{1}}\left\{1-\cos \left[2\left(\varphi+\beta_{1}\right)\right]\right\} \\
& -\frac{\varepsilon \cos \left(\varphi+\beta_{1}\right)}{\gamma_{1} a_{1}} \times\left[p_{2} \Omega^{2} \cos (\varphi)+\mu_{1} a_{1} \omega_{1} \sin \left(\varphi+\beta_{1}\right)\right] \\
& -\frac{\varepsilon p_{5}}{\gamma_{1} a_{1}} \sum_{k=1}^{10} G_{k}^{\prime}+O\left(\varepsilon^{2}\right) \\
\Omega^{\prime}= & \varepsilon\left[M_{1}(\Omega)-p_{3} a_{1} \omega_{1} \Omega \cos \left(\varphi+\beta_{1}\right) \sin (\varphi)-\frac{p_{4} g \cos (\varphi)}{\omega^{2}}\right] \\
& +O\left(\varepsilon^{2}\right) \\
a_{3}^{\prime}= & \sum_{i=1}^{7} J_{i} \\
\beta_{3}^{\prime}= & \sum_{k=1}^{8} J_{k}
\end{aligned}
$$

where $G_{i}, G_{k}^{\prime}, J_{i}$ and $J_{k}$ depend on sines and/or cosines with frequency, phases and amplitudes determined by an algebraic expansion (see Appendix).

Introducing the detuning parameter to express the difference of the rotation frequency of the motor with the oscillation frequency of the block, $\Omega=1+\varepsilon \sigma_{\Omega}$ [31], results:

$-\Omega+\frac{\omega_{1}^{2}}{\gamma}=-\frac{\varepsilon\left(\sigma_{\Omega}+p_{5} \delta\right)}{\gamma}+O\left(\varepsilon^{2}\right)$

Given that the sine and cosine functions are limited, and $a_{1}^{\prime}, \beta_{1}^{\prime}, a_{3}^{\prime}, \beta_{3}^{\prime}, \Omega$ are functions of order $\varepsilon, \varepsilon$ being a small parameter, then the functions slowly vary over $\tau$, and can be considered constants in the interval $[0,2 \pi][2]$.

According to Nayfeh [2] there are two ways of doing the calculus of the average. The first is to calculate the average of the Eq. (15) over a period of the circular functions, to determinate a first approximation. In the second method, only the terms of slow variation are kept, and to the first approximation, the average of the equations are considered to be the slow varying terms. In this work, the second method is adopted, and the case without resonance between the block and the pendulum, but with primary resonant interaction between the pendulum and the motor is analyzed. Nayfeh [2] referred this condition is his studies as the non-resonant case. 
Considering the system far from the resonance, there will be a resonant interaction only between the block and the motor. Averaging the Eq. (15), the system that describes the behavior of the system far from the resonance, is obtained.

$$
\begin{aligned}
a_{1}^{\prime}= & -\frac{\varepsilon}{2 \gamma}\left(p_{2} \Omega^{2} \sin \beta_{1}+\mu_{1} a_{1}\right) \\
\beta_{1}^{\prime}= & -\varepsilon \frac{\left(a_{1}+p_{5} \delta\right)}{\gamma}+\varepsilon \frac{p_{5} \delta \omega_{1}}{2 \gamma}-\varepsilon \frac{p_{2} \Omega^{2}}{2 \gamma a_{1}} \cos \beta_{1}-\frac{\varepsilon p_{5}}{2 \gamma a_{1}} \\
& \times\left(\frac{a_{3}^{2} \omega_{3} a_{1} \delta}{2}+\frac{a_{3}^{3} \omega_{1}^{2} \delta^{3}}{2}-\frac{a_{1}^{3} \omega_{1}^{2} \delta^{3}}{4}\right) \\
\Omega^{\prime}= & \varepsilon\left[M_{1}(\Omega)-\frac{1}{2} p_{3} a_{1} \omega_{1} \Omega \sin \left(-\beta_{1}\right)\right] \\
a_{3}^{\prime}= & -\varepsilon \frac{\mu_{3} a_{3}}{2} \\
\beta_{3}^{\prime}= & 0
\end{aligned}
$$

To determine the responses of the steady states, there is no need to integrate numerically the set of averaged equations describing the amplitudes and phases of the system. Instead, it is considered that $a_{1}, \beta_{1}, a_{3}, \beta_{3}$ and $\Omega$ are constants in the steady state and, then, the derivatives that describe the modulations of amplitude and phase are null [2], resulting in the following set of algebraic equations:

$$
\begin{aligned}
& -\frac{\varepsilon}{2 \gamma}\left[p_{2} \Omega^{2} \sin \left(\beta_{1}\right)+\mu_{1} a_{1}\right]=0 \\
& -\frac{\varepsilon\left(\alpha_{1}+p_{5} \delta\right)}{\gamma}+\frac{\varepsilon p_{5} \delta \omega_{1}}{2 \gamma} \\
& -\frac{\varepsilon p_{2} \Omega^{2}}{2 \gamma a_{1}} \cos \left(\beta_{1}\right)-\frac{\varepsilon p_{5}}{2 \gamma a_{1}}\left(\frac{a_{3}^{2} \omega_{3} a_{1} \delta}{2}+\frac{a_{3}^{3} \omega_{1}^{2} \delta^{3}}{2}-\frac{a_{1}^{3} \omega_{1}^{2} \delta^{3}}{4}\right)=0 \\
& \varepsilon\left[M_{1}(\Omega)-p_{3} a_{1} \omega_{1} \Omega \frac{1}{2} \sin \left(-\beta_{1}\right)\right]=0 \\
& -\varepsilon \frac{\mu_{3} a_{3}}{2}=0
\end{aligned}
$$

Taking into account the fourth equation and combining the first and the second equations in (18) yields:

$$
\left[\mu_{1}^{2}+\left(2 \sigma+p_{5} \delta-\frac{p_{5} \delta^{3} a_{1}^{2}}{4}\right)^{2}\right] a_{1}^{2}=\left(p_{2} \Omega^{2}\right)^{2}
$$

Combining the first and the third equations yields:

$$
M_{1}(\Omega)-\frac{p_{3} \mu_{1} a_{1}^{2}}{2 p_{2} \Omega}=0
$$

The amplitude of the electro-mechanic pendulum motion, and the rotational frequency of the motor are determined from (19) and (20), respectively, for each value of the control parameter $\Omega$ [32]. Additionally, the phase of motion in the steady-state response is given by:

$$
\tan \left(\beta_{1}\right)=-\frac{\mu_{1}}{2\left(\frac{p_{5} \delta^{3} a_{1}^{2}}{8}-2 \sigma-p_{5} \delta\right)}
$$

The Eq. (12) is similar to the equation obtained and analyzed by [32] using the multiple scales method, and the averaging method. To determinate the stability of the steady states $a_{1 c}, \beta_{1 c}, a_{3 c}, \beta_{3 c}$ and $\Omega_{c}$, the same technique of [32] is applied, considering only the first four equations in (18), and introducing a small perturbation, yielding:

$a_{1}=a_{1 c}+\varepsilon A_{1} ; \beta_{1}=\beta_{1 c}+\varepsilon B_{1} ;$

$a_{3}=a_{3 c}+\varepsilon A_{3} ; \Omega=\Omega_{c}+\varepsilon \Omega_{1}$

Replacing the small perturbations in the system and expanding in Taylor series around the stationary states, and keeping only the linear terms, results:

$\mathbf{Y}^{\prime}=\left.\mathbf{J}\right|_{\mathbf{Y} *} \mathbf{Y}$

where $\mathbf{Y}=\left[\begin{array}{llll}\Omega_{1} & A_{1} & B_{1} & A_{3}\end{array}\right]^{T}, \mathbf{Y}^{*}=\left[\begin{array}{llll}\Omega_{c} & a_{1 d} & \beta_{1 c} & a_{3 c}\end{array}\right]^{T}$. The Jacobian matrix $\mathbf{J}=\left[b_{i j}\right]$ is given by:

$\mathbf{J}=\left[\begin{array}{cccc}\varepsilon\left(N-\frac{p_{3} \mu_{1} a_{1 c}^{2}}{2 p_{2} \Omega_{c}^{2}}\right) & -\varepsilon\left(\frac{p_{3} \mu_{1} a_{1 c}}{2 p_{2} \Omega_{c}}\right) & b_{13} & 0 \\ \frac{\varepsilon \mu_{1} a_{1 c}}{\gamma \Omega_{c}} & \frac{-\varepsilon \mu_{1}}{2 \gamma} & b_{23} & 0 \\ b_{31} & b_{32} & \frac{-\varepsilon \mu_{1}}{2 \gamma} & b_{34} \\ 0 & 0 & 0 & \frac{-\varepsilon \mu_{3}}{2}\end{array}\right]$

With $\quad b_{13}=-\varepsilon \frac{p_{3} a_{1 c}}{p_{2}}\left[\left(\sigma_{\Omega}+\frac{p_{5} \delta}{2}\right) \frac{a_{1}}{\Omega}+\frac{p_{5} \delta_{2}}{2 \Omega_{c}}\right]$, $b_{23}=\frac{\varepsilon a_{1 c}}{\gamma}\left[\left(\sigma_{\Omega}+\frac{p_{5} \delta}{2}\right)+\frac{p_{5} \delta_{2}}{2 a_{1 c}}\right]$,

$b_{31}=\frac{2 \varepsilon}{\gamma a_{1 c}}\left[\left(\sigma_{\Omega}+\frac{p_{5} \delta}{2}\right) \frac{a_{1 c}}{\sigma_{\Omega}}+\frac{p_{5} \delta_{2}}{2 \sigma_{\Omega}}\right]$,

$b_{32}=\frac{-\varepsilon}{\gamma a_{1 c}}\left[\left(\sigma_{\Omega}+\frac{p_{5} \delta}{2}\right)+\frac{p_{5}}{2} \frac{\partial}{\partial a_{1 c}}\left(\delta_{2}\right)\right]$,

$b_{34}=\frac{-\varepsilon p_{5}}{2 \gamma a_{1 c}}\left(a_{3 c} \omega_{3} \delta a_{1 c}+\frac{3 a_{3 c}^{2} \delta^{3}}{2}\right), N=\frac{\partial}{\partial \Omega}\left(M_{1}(\Omega)\right)$, and

$\delta_{2}=\left(\frac{a_{3 c}^{2} \omega_{3} a_{1 c} \delta}{2}+\frac{a_{3 c}^{3} \delta^{3}}{2}-\frac{a_{1 c}^{3} \delta^{3}}{4}\right)$.

The roots of the characteristic polynomial are given by:

$\left(\varepsilon \frac{\mu_{3}}{2}+\lambda\right)\left(\lambda^{3}-S_{1} \lambda^{2}+S_{2} \lambda-S_{3}\right)=0$

where

$$
\begin{aligned}
S_{1}= & b_{11}+b_{22}+b_{33} \\
S_{2}= & \left(b_{22} b_{33}-b_{32} b_{23}\right) \\
& +\left(b_{11} b_{33}-b_{13} b_{31}\right)+\left(b_{11} b_{22}-b_{12} b_{21}\right)
\end{aligned}
$$

$S_{3}=\operatorname{det}(\mathbf{J})$

According to the Routh-Hurwitz criterion [33], the necessary and sufficient conditions to the roots of the polynomial to have negative real parts, are: $-S_{1}>0 ;-S_{3}>0$; $S_{1} S_{2}-S_{3}>0$. These conditions are used to obtain the number of eigenvalues with positive real parts. 


\section{Steady state}

In this section the dynamical system is analyzed considering different control parameters, curves with hysteresis effect, curves of hardening and softening types and jumps in the amplitudes of the motions, are obtained.

\subsection{Parameter of control: frequency $\Omega$ of the motor rotation}

Considering $\Omega$ as a control parameter, Eq. (19) is an implicit equation of the amplitude $a_{1}$ and a function of the angular frequency $\Omega$. Figure $2 \mathrm{~b}$ shows the characteristic curve for $a=0.05, \quad b=0.12, \quad m_{1}=3.850$ $(\mathrm{kg}), m_{2}=0.008532(\mathrm{~kg}), m_{3}=0.5(\mathrm{~kg}), l=1.19(\mathrm{~m})$, $R=0.075 \quad(\mathrm{~m}), \quad c_{1}=0.0008622 \quad(\mathrm{Nm} / \mathrm{s}), \quad c_{3}=0.3234$ $(\mathrm{Nm} / \mathrm{s}), \quad k_{1}=1.75 \quad(\mathrm{~N} / \mathrm{m}), \quad \sigma=-0.00099, \quad \Omega_{1}=0.1$, $\Omega_{f}=55.4, a_{3}=0.009, J_{2}=0.37\left(\mathrm{~kg} \mathrm{~m}^{2}\right)$. This diagram shows the stable and unstable stationary states for the averaging Eq. (17).

The stable stationary states are $0^{\wedge} \Omega_{c 1}$ and $\Omega_{c 2}^{\wedge} \Omega_{c}$, and the unstable stationary states are $\Omega_{c 1}^{\wedge} \Omega_{c 2}$ and for $\Omega>45.16$. In $\Omega_{c 1}^{\wedge} \Omega_{c 2}$ the instability is due to the appearance of a positive eigenvalue for $\Omega=9.97$ and for $\Omega>19.72$. For $\Omega=45.16$ the instability is due to the appearing of two eigenvalues with positives real parts.

According to [2], the characteristics of the curve of frequency-response are sufficient to characterize the jump effect, that is, in the points where the tangent of a curve become vertical, and a jump in the amplitude of the movements occur.

More precisely, the amplitude of the oscillations increase over the control parameter $\Omega$, reaching a critical value $\Omega_{c 1}$, shown in Fig. 2a. For $\Omega=19.72$ there is a jump in the amplitude of oscillation, see point 1 , and continue to follow up until reach the point $\Omega>45.16$, where the system becomes unstable due to the appearance of two eigenvalues with positive real parts.

On the other side, following the decreasing direction of $\Omega$, the system is unstable $\Omega>45.16$, and for $\Omega<45.16$ the oscillation amplitude of the block decreases until reaching $\Omega=\Omega_{c 2}$, where the system experiments a new jump to the point 2 shown in Fig. 2a and, continues softly decreasing until reaching $\Omega=0$.

For $8.97<\Omega<19.72$, the frequency-response curve depends on whether $\Omega$ is increasing or decreasing. This phenomenon is called hysteresis [2].

It can be noticed that the system is strongly dependent on the power source dynamics. For $b=0.07$ the system becomes unstable behavior at the point $\Omega=27.61$ (Fig. 2b). When $b=0.02$ the system becomes unstabe for $\Omega=13.19$ (Fig. 2c).
Forced oscillations are possible only if Eq. (13) is satisfied. In Fig. 2d it can be seen that for a given amplitude of oscillations, up to three roots can be found [1].

Additional modifications on the power source characteristics and on the torque parameters, for example, for $a=0.03$ and $b=0.001$, generates much more complex behaviors, indicating the need for further studies to explain the electro-mechanical pendulum system dynamics.

\subsection{Control parameter: detuning parameter $\sigma_{\Omega}$, and $\delta>0$}

Considering $\sigma_{\Omega}$ as a control parameter, the curves with softening and hardening characteristics are obtained, depending on whether $\delta$ is positive or negative. For $\delta>0$, the curve is hardening, otherwise, the curve is softening.

In Fig. 3, the characteristics curves are presented for: $m_{1}=3.30(\mathrm{~kg}), m_{2}=0.002(\mathrm{~kg}), m_{3}=0.15(\mathrm{~kg}), l=1.19$ $(\mathrm{m}), R=0.075(\mathrm{~m}), c_{1}=0.000898622(\mathrm{Nm} / \mathrm{s}), c_{3}=0.234$ $(\mathrm{Nm} / \mathrm{s}), k_{1}=2.2(\mathrm{~N} / \mathrm{m}), \sigma_{\Omega_{0}}=-0.004, \sigma_{\Omega_{f}}=0.0015$, $a_{3}=0.09, a=0.01, b=0.0009$, and $J_{2}=0.37\left(\mathrm{~kg} \mathrm{~m}^{2}\right)$.

In Figs. 3a, b and 4c the cases for $\Omega=25.3, \Omega=10.725$ and $\Omega=9.75$, are respectively shown. In Fig. $3 \mathrm{~b}$ the instability region is due to a positive eigenvalue, while in Fig. $3 \mathrm{c}$ a region of jump in the amplitudes of motion is shown.

\subsection{Control parameter: detuning parameter $\sigma_{\Omega}$, and $\delta<0$}

For $\delta<0$ Fig. 4 represents a characteristic curve for the control parameter $\sigma_{\Omega}$ and $m_{1}=1.30(\mathrm{~kg}), m_{2}=0.01(\mathrm{~kg}), m_{3}=0.3$ $(\mathrm{kg}), l=1.19(\mathrm{~m}), R=0.075(\mathrm{~m}), c_{1}=0.8622(\mathrm{Nm} / \mathrm{s})$, $c_{3}=1.3234(\mathrm{Nm} / \mathrm{s}), k_{1}=35.2(\mathrm{~N} / \mathrm{m}), \sigma_{0}=-0.5, \sigma_{f}=0.8$, $a_{3}=0.09, a=0.19, b=0.00051, J_{2}=0.4\left(\mathrm{~kg} \mathrm{~m}^{2}\right)$.

Figure $4 \mathrm{a}-\mathrm{c}$ show the cases for $\Omega=25.3, \Omega=18$ and $\Omega=17$, respectively. In Fig. $4 \mathrm{~b}$, the unstable points on the left side are due to two positive eigenvalues, and the unstable points on the right side are due to a positive eigenvalue. The jump effect can be seen in Fig. 4c.

The jump phenomenon is characterized by a sudden amplitude transition, as indicated in Figs. $3 \mathrm{c}$ and $4 \mathrm{c}$. This happens because there is not enough damping in the system to stop the DC motor from transmitting large amounts of energy to the nonlinear oscillator [11].

In Figs. 3 and 4 the influence of parameter $(\delta)$ in the curve type ("softening or hardening") can be observed. Similar results were obtained for the non-ideal model in [1].

Non-ideal sources present a strong dependence on the energy supply characteristics, and small changes in the power supply, imply modifications on the stability characteristics of the system. This behavior can be seen 

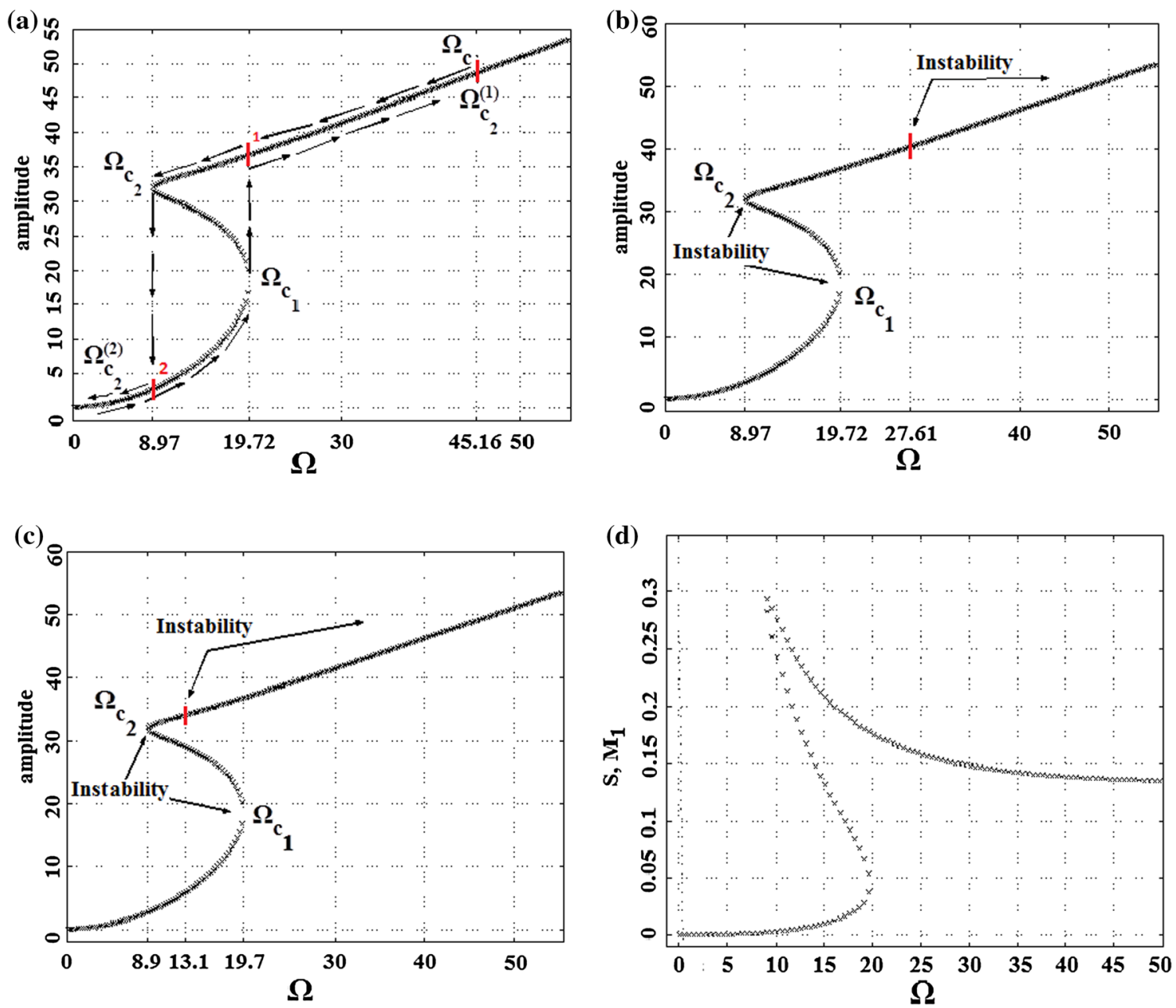

Fig. 2 a Frequency-response curve and hysteresis phenomenon, $\mathrm{b}=0.12$. b Unstable and stable points for $b=0.07$. c Unstable and stable points for $b=0.02$. d Intersection of the curves $S(\Omega)$ and linear torque $M_{1}(\Omega)$

in Figs. 2b, c, 3b and 4b. Figures 2b, c, 3 b and $4 b$ show unstable points for variations of the control parameter $(\Omega)$ and $\left(\sigma_{\Omega}\right)$, whereas Figs. $3 \mathrm{c}$ and $4 \mathrm{c}$ show that the jumps in the achievable amplitude movements do not occur near the vertical axis, making evident the effects of non-ideal source on the system behavior $[1,8-12$, 31].

In Fig. 5 a comparison of the system (3) and its approximation by average method (Eqs. (6) and (7)) is shown, considering $a=0.05, b=0.12, m_{1}=3.850(\mathrm{~kg})$, $m_{2}=0.008532(\mathrm{~kg}), m_{3}=0.5(\mathrm{~kg}), l=1.19(\mathrm{~m}), R=0.075$ (m), $c_{1}=0.0008622(\mathrm{Nm} / \mathrm{s}), c_{3}=0.3234(\mathrm{Nm} / \mathrm{s}), k_{1}=1.75$ $(\mathrm{N} / \mathrm{m}), \sigma=-0.00099, \Omega_{1}=0.1, \Omega_{f}=55.4, a_{3}=0.009$, $J_{2}=0.37(\mathrm{~kg} \mathrm{~m}), \quad a_{1}=1.1, \quad a_{3}=0, \beta_{1}=-0.1629 \tau$, $\beta_{3}=0, \Omega=0.7416$ and $\delta=0.0513$.

\section{Application of the SDRE control}

To control $u, u^{\prime}, \theta$ and $\theta^{\prime}$, forcing the system to the orbit of Eq. (6), the variables $\varphi$ and hedynamicalelectromechanicpendulumsystemequa are considered as disturbances. Then, the dynamic system defined of Eq. (3) can be parameterized as a first order state space equation, written in the state-dependent coefficient (SDC) and non-statedependent coefficient:

$\mathbf{x}^{\prime}=\mathbf{A}_{\mathbf{x}} \mathbf{x}+\mathbf{B} \mathbf{U}+\mathbf{F}\left(x_{i}\right)$

where $\quad \mathbf{x}=\left[\begin{array}{llll}x_{1} & x_{2} & x_{2} & x_{3}\end{array}\right]^{T}$ is the state vector, $\mathbf{x}^{\prime}$ is the vector of the first order derivatives. $\mathbf{U}=\left[\begin{array}{llllll}0 & u_{s f 1}+\tilde{u}_{s 1} & 0 & u_{s f 2}+\tilde{u}_{s 2}\end{array}\right]^{T}, \quad$ where $\quad \mathbf{u}_{s f}$ is the 

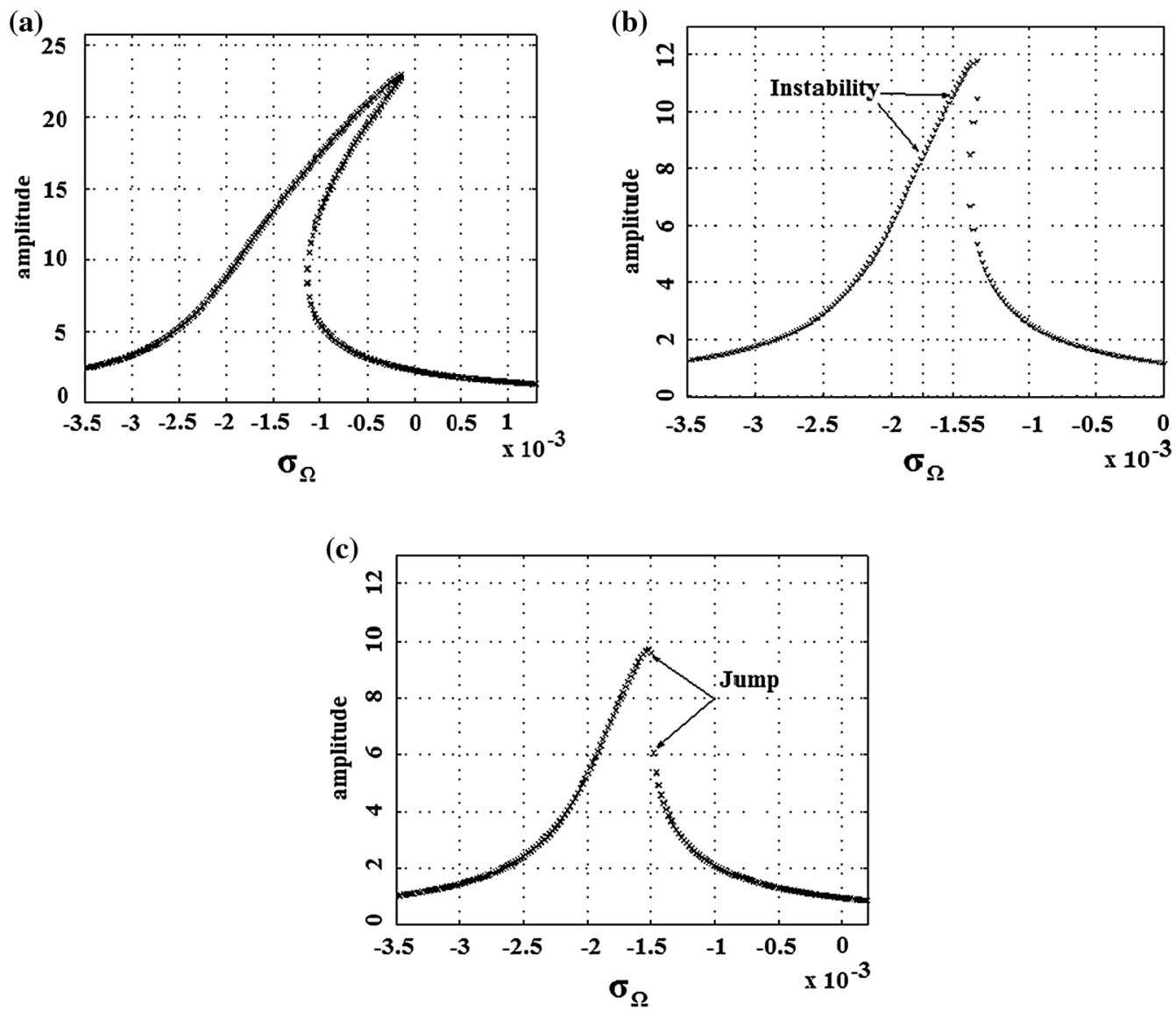

Fig. 3 Frequency-response curve as a function of the control parameter $\sigma_{\Omega}$ and $\delta>0$. a Frequency- response curve for $\delta>0$ and $\Omega=25.3$. b Frequency-response curve for $\delta>0$ and $\Omega=10.725$. c Frequency-response curve with $\delta>0$ and $\Omega=9.75$

feedback control signal, $\tilde{\mathbf{u}}_{s}$ is the feedforward control signal, and $\boldsymbol{F}$ is the nonlinear vector, $i=1,2, \ldots, 6, x_{1}=u$, $x_{2}=u^{\prime}, x_{3}=\theta, x_{4}=\theta^{\prime}, x_{5}=\varphi$ and $x_{6}=\varphi^{\prime}$.
Considering the system of Eq. (3) written in the form of Eq. (27), results:

$$
\begin{gathered}
\mathbf{A}_{\mathbf{x}}=\left[\begin{array}{ccc}
0 & 1 & 0 \\
-\Phi-\Phi \varepsilon \mu_{1} & -\Phi \varepsilon p_{5}\left(\omega_{3}^{2}+x_{4}^{2}\right) & -\Phi \varepsilon p_{5} \varepsilon \mu_{3} \\
\Phi & \Phi \varepsilon \mu_{1} & -\omega_{3}^{2}+\Phi \varepsilon p_{5}\left(x_{4}^{2}+\omega_{3}^{2}\right) \\
0 & -\varepsilon \mu_{3}\left(1-\Phi \varepsilon p_{5}\right)
\end{array}\right], \\
\mathbf{F}\left(x_{i}\right)=\left[\begin{array}{c}
\Phi\left(\begin{array}{c}
\frac{\varepsilon \alpha}{\omega^{2}} \varepsilon p_{2} \sin \left(x_{5}\right)-\frac{\varepsilon \beta}{\omega} \varepsilon p_{2} \sin \left(x_{5}\right) x_{6} \\
+\varepsilon p_{2} x_{6}^{2} \cos \left(x_{5}\right)-\frac{g}{\omega^{2}} \varepsilon p_{2} \varepsilon p_{4} \sin \left(x_{5}\right) \cos \left(x_{5}\right)
\end{array}\right) \\
0 \\
-\Phi\left(\begin{array}{c}
\frac{\varepsilon \alpha}{\omega^{2}} \varepsilon p_{2} \sin \left(x_{5}\right)-\frac{\varepsilon \beta}{\omega} \varepsilon p_{2} \sin \left(x_{5}\right) x_{6} \\
-\frac{g}{\omega^{2}} \varepsilon p_{2} \varepsilon p_{4} \sin \left(x_{5}\right) \cos \left(x_{5}\right)+\varepsilon p_{2} x_{6}^{2} \cos \left(x_{5}\right)
\end{array}\right)
\end{array}\right], \quad \mathbf{B}=\left[\begin{array}{ll}
0 & 0 \\
1 & 0 \\
0 & 0 \\
0 & 1
\end{array}\right],
\end{gathered}
$$


(a)

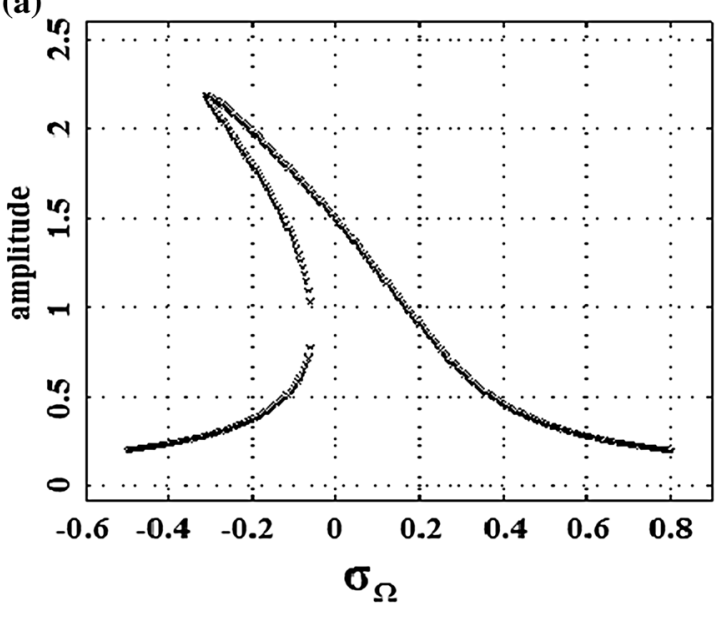

(b)

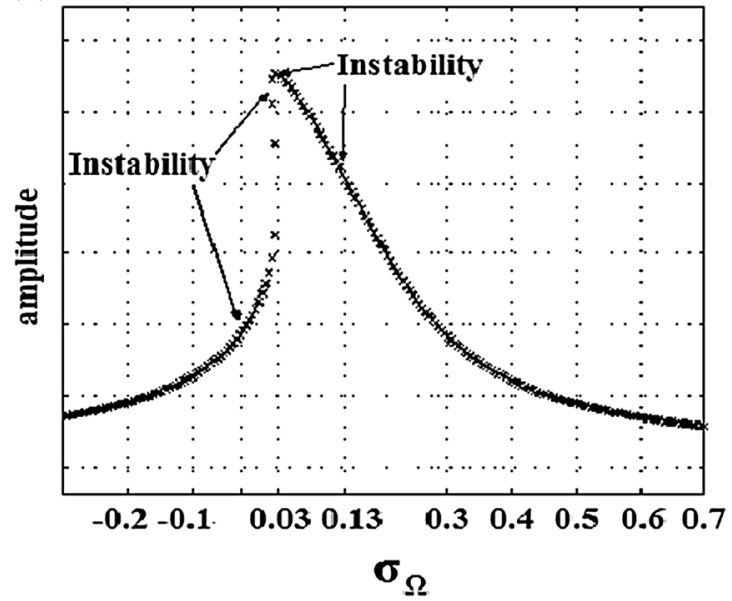

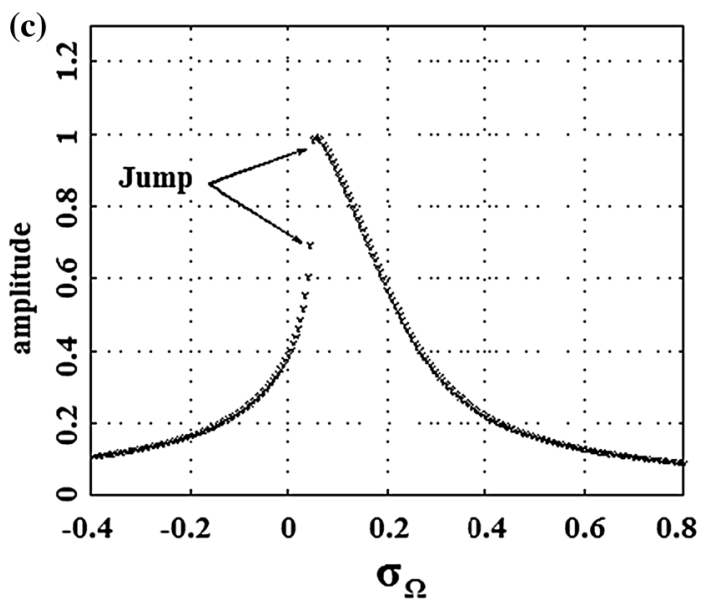

Fig. 4 a Frequency-response curve for $\delta<0$ and $\Omega=25.3$. b Frequency-response curve with $\delta<0$ and $\Omega=18$. c Frequency-response curve with $\delta<0$ and $\Omega=17$

Fig. 5 System (3) and approximation by Eqs. (6) and (7): a phase diagram for horizontal displacement. b Phase diagram for the angular displacement of the pendulum

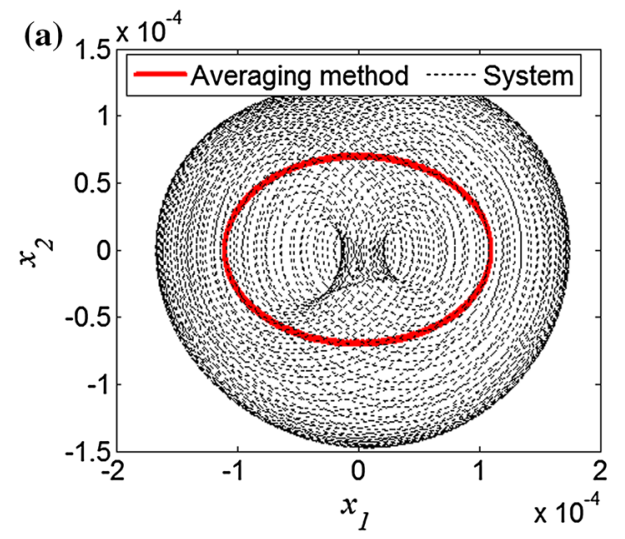


Fig. 6 System (3) with control: a phase diagram for horizontal displacement. b Phase diagram for the angular displacement of the pendulum

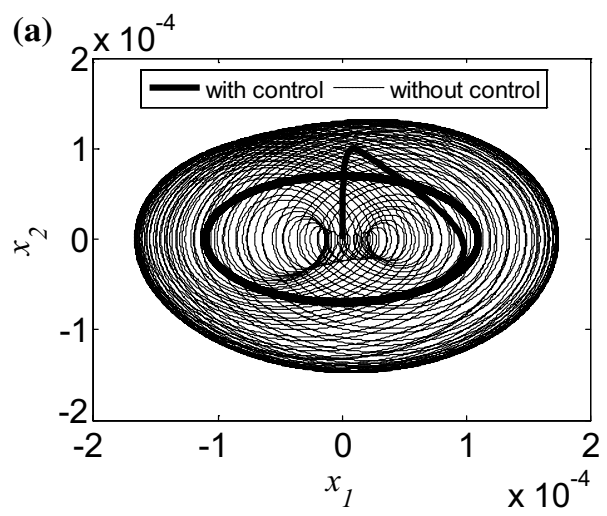

where: $\Phi=\frac{1}{\varepsilon p_{5}-\varepsilon p_{2} \varepsilon p_{3} \sin \left(x_{5}\right)^{2}}$, and $x_{5}, x_{6}$ are given by:

$$
\begin{aligned}
x_{5}^{\prime}= & x_{6} \\
x_{6}^{\prime}= & \frac{\varepsilon \alpha}{\omega^{2}}-\frac{\varepsilon \beta}{\omega} x_{6}-\frac{\varepsilon p_{4} g}{\omega^{2}} \cos \left(x_{5}\right)+\varepsilon p_{3} \sin \left(x_{5}\right) \Phi \\
& \times\left(\begin{array}{l}
-\varepsilon \mu_{1} x_{2}-x_{1}+\frac{\varepsilon \alpha}{\omega^{2}} \varepsilon p_{2} \sin \left(x_{5}\right) \\
-\varepsilon p_{5} x_{4}^{2} x_{3}-\varepsilon p_{5} \varepsilon \mu_{3} x_{4}-\varepsilon p_{5} \omega_{3}^{2} x_{3} \\
-\frac{g}{\omega^{2}} \varepsilon p_{2} \varepsilon p_{4} \sin \left(x_{5}\right) \cos \left(x_{5}\right) \\
+\varepsilon p_{2} x_{6}^{2} \cos \left(x_{5}\right)-\frac{\varepsilon \beta}{\omega} \varepsilon p_{2} \sin \left(x_{5}\right) x_{6}
\end{array}\right)
\end{aligned}
$$

A state feedback instead of output feedback is adopted to improve the control performance. The cost function for the regulator problem is given by:

$J_{\mathrm{UF}}=\frac{1}{2} \int_{t_{0}}^{\infty}\left[\mathbf{y}^{T} \mathbf{Q y}+\mathbf{u}_{s f}^{T} \mathbf{R} \mathbf{u}_{s f}\right] \mathrm{d} \tau$

where: $\mathbf{y}=\left[x_{1}-x_{1}^{*} x_{2}-x_{2}^{*} x_{3}-x_{3}^{*} x_{4}-x_{4}^{*}\right]^{T}, x^{*}$ are the desired states, $\mathbf{Q}$ is a semi-positive-definite matrix and $R$ positive definite. Assuming full state feedback, the control law is given by:

$\mathbf{u}_{\mathrm{sf}}=-\mathbf{R}^{-1} \mathbf{B}^{T} \mathbf{P}_{x} \mathbf{y}$

where: $\mathbf{P}_{\mathbf{X}}$ is obtained by the solution of the state-dependent Riccati equation, given by:

$\mathbf{A}_{\mathbf{x}}^{T} \mathbf{P}_{\mathbf{x}}+\mathbf{P}_{\mathbf{x}} \mathbf{A}_{\mathbf{x}}-\mathbf{P}_{\mathbf{x}} \mathbf{B}_{\mathbf{x}} \mathbf{R}^{-\mathbf{1}} \mathbf{B}^{\mathbf{T}} \mathbf{P}_{\mathbf{x}}+\mathbf{Q}=\mathbf{0}$

The applied to feedback control (30) implies in the minimization of the functional (29) and in the minimization of the system deviation $(\mathbf{y})$, of the desired state $\left(\mathbf{x}^{*}\right)$.

Defining the feedforward control as:

$$
\begin{aligned}
& \tilde{u}_{s 1}=-\Phi\left(\begin{array}{l}
\frac{\varepsilon \alpha}{\omega^{2}} \varepsilon p_{2} \sin \left(x_{5}\right)-\frac{\varepsilon \beta}{\omega} \varepsilon p_{2} \sin \left(x_{5}\right) x_{6} \\
+\varepsilon p_{2} x_{6}^{2} \cos \left(x_{5}\right)-\frac{g}{\omega^{2}} \varepsilon p_{2} \varepsilon p_{4} \sin \left(x_{5}\right) \cos \left(x_{5}\right)
\end{array}\right) \\
& \tilde{u}_{s 2}=\Phi\left(\begin{array}{l}
\frac{\varepsilon \alpha}{\omega^{2}} \varepsilon p_{2} \sin \left(x_{5}\right)-\frac{\varepsilon \beta}{\omega} \varepsilon p_{2} \sin \left(x_{5}\right) x_{6} \\
-\frac{g}{\omega^{2}} \varepsilon p_{2} \varepsilon p_{4} \sin \left(x_{5}\right) \cos \left(x_{5}\right)+\varepsilon p_{2} x_{6}^{2} \cos \left(x_{5}\right)
\end{array}\right)
\end{aligned}
$$

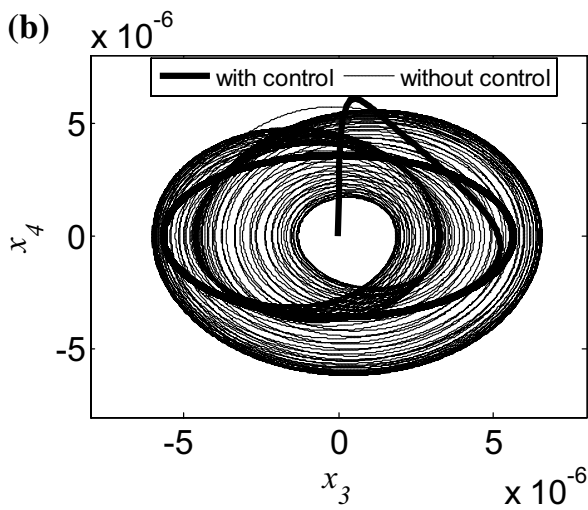

Replacing (32) into (27), the system can be represented in the form:

$\mathbf{x}^{\prime}=\mathbf{A}_{\mathbf{x}} \mathbf{x}+\mathbf{B} \mathbf{u}_{s f}$

\section{Numerical simulation of the proposed control}

Considering the parameters: $a=0.05, b=0.12, m_{1}=3.850$ $(\mathrm{kg}), m_{2}=0.008532(\mathrm{~kg}), m_{3}=0.5(\mathrm{~kg}), l=1.19(\mathrm{~m})$, $R=0.075 \quad(\mathrm{~m}), \quad c_{1}=0.0008622(\mathrm{~N} \mathrm{~m} / \mathrm{s}), \quad c_{3}=0.3234$ $(\mathrm{N} \mathrm{m} / \mathrm{s}), \quad k_{1}=1.75(\mathrm{~N} / \mathrm{m}), \quad \sigma=-0.00099, \quad \Omega_{1}=0.1$, $\Omega_{f}=55.4, a_{3}=0.009, J_{2}=0.37\left(\mathrm{~kg} \mathrm{~m}^{2}\right)$.

And defining the desired states as (6 and 7):

$x_{1}^{*}=a_{1} \cos \left(x_{5}+\beta_{1}\right)$

$x_{2}^{*}=-a_{1} \Omega \sin \left(x_{5}+\beta_{1}\right)$

$x_{3}^{*}=a_{3} \cos \left(\omega_{3} \tau+\beta_{3}\right)+a_{1} \delta \cos \left(x_{5}+\beta_{1}\right)$,

$x_{4}^{*}=-a_{3} \omega_{3} \sin \left(\omega_{3} \tau+\beta_{3}\right)-a_{1} \Omega \delta \sin \left(x_{5}+\beta_{1}\right)$

Considering Eqs. (17) the following parameters for (34) are defined as $a_{1}=1.1, a_{3}=0, \beta_{1}=-0.1629 \tau, \beta_{3}=0$, $\Omega=0.7416$ and $\delta=0.0513$.

Defining the matrices: $\mathbf{Q}=10^{3}[\mathbf{I}]_{4 \times 4}$ and $\mathbf{R}=[\mathbf{I}]_{2 \times 2}$, the dynamics of the controlled system (27) can be seen in Fig. 6 .

It can observed that the proposed control is efficient in taking the states (x) of the system (27) to the desired states $\mathbf{x}^{*}(34)$.

\section{Control system behavior in the presence of parametric errors}

To test the effect of parameter uncertainties on the performance of the controller $\mathbf{u}_{\mathrm{sf}}$, the parameters $\varepsilon \mu_{1}, \varepsilon p_{5}, \varepsilon \mu_{3}$ and $\omega_{3}$ used in matrix $\left(\mathbf{A}_{x}\right)$ are considered with a random error of $\pm 20 \%[21,27,28,30]$.

In Fig. 7 the variation of system (27) with (control without parametric error) and with (control with parametric error) can be seen, and the SDRE control strategy proved 
Fig. 7 Variation of error for $u$, $u^{\prime}, \theta$ and $\theta^{\prime}$ for (control without parametric error) and (control with parametric error)
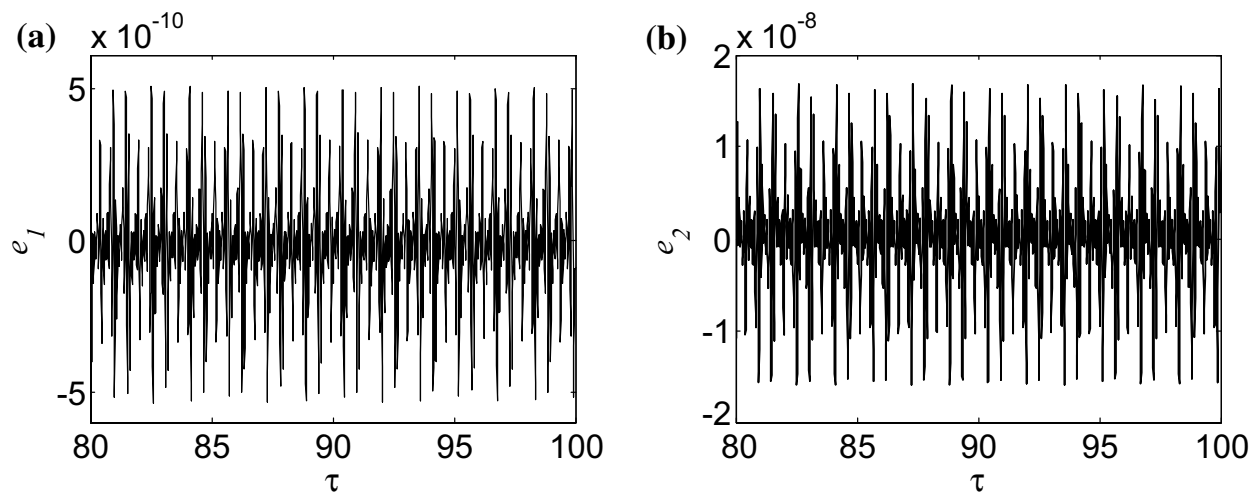

(c)

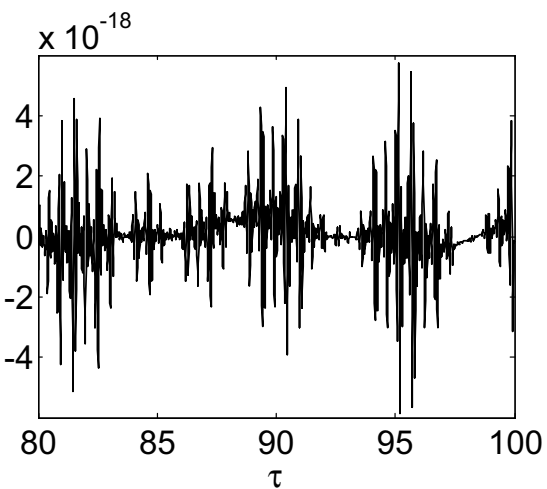

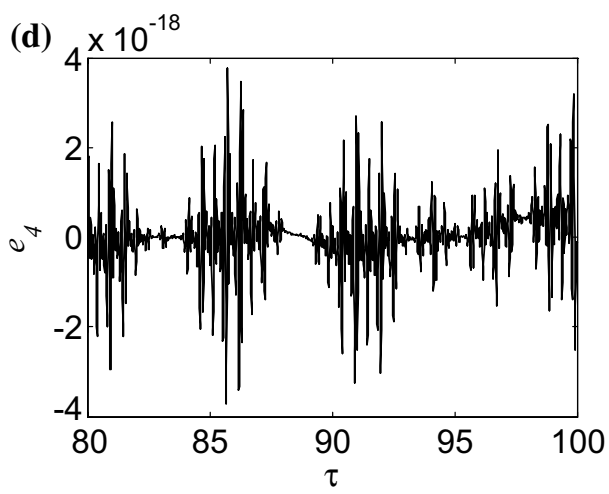

to be robust in the case of parametric variations with errors smaller than $\left(2 \times 10^{-8}\right)$.

\section{Conclusion}

In this work the dynamical behavior of the electro-mechanical pendulum is investigated, applying the SDRE control to synchronize the system motion in a periodic orbit. This system presents complex dynamical effects such as hysteresis, curves of softening and hardening type and Sommerfeld effects. The Routh-Hurwitz criterion is used to determine the steady states stability. Analytical solutions are not feasible due to the complexity of the system's equations. Then some simplifications have been considered enabling the use of average methods $[8,31]$. The curves obtained from the frequency response equation show that the electro-mechanical pendulum system presents rich dynamical behavior, exhibiting similar results to that obtained by [8-12, 31]. As it can be seen in Fig. 5a, b, the proposed feedforward control $\left(\mathbf{u}_{s f}\right)$ and a feedback $\left(\tilde{\mathbf{u}}_{s}\right)$ allow to control the oscillations of the electromechanical pendulum system in the periodic orbits obtained by averaging method. Additionally, it can be concluded that the proposed feedback control based on the SDRE method is robust to parametric uncertainties. Similar results are shown in $[21,27,28,30]$. Showing that the SDRE control strategy is indicated for the electromechanical pendulum control problem.
Acknowledgments The authors would like to acknowledge São Paulo Research Foundation-FAPESP (Grant: 2013/04101-6), and Conselho Nacional de Desenvolvimento Científico e Tecnológico-CNPQ (Grant: 447539/2014-0 and 303903/2014-7) for the financial support.

\section{Appendix}

$$
\begin{aligned}
J_{1}= & \frac{a_{1}^{\prime} \omega_{1}}{2 \omega_{3}}\left\{\cos \left[\left(\Omega-\omega_{3}\right) \tau+\left(\beta_{1}-\beta_{3}\right)\right]\right. \\
& \left.-\cos \left[\left(\Omega-\omega_{3}\right) \tau+\left(\beta_{1}+\beta_{3}\right)\right]\right\} \\
J_{2}= & \frac{a_{1} \beta_{1}^{\prime} \omega_{1}}{2 \omega_{3}}\left\{\sin \left[\left(\Omega+\omega_{3}\right) \tau+\left(\beta_{1}+\beta_{3}\right)\right]\right. \\
& \left.-\sin \left[\left(\Omega-\omega_{3}\right) \tau+\left(\beta_{1}-\beta_{3}\right)\right]\right\} \\
J_{3}= & -\frac{a_{3} \mu_{3}}{2}\left[1-\cos \left(2 \omega_{3} \tau+2 \beta_{3}\right)\right] \\
J_{4}= & -\varepsilon \frac{\delta a_{1} \omega_{1} \mu_{3}}{2 \omega_{3}}\left\{\cos \left[\left(\Omega-\omega_{3}\right) \tau+\left(\beta_{1}-\beta_{3}\right)\right]\right. \\
& \left.-\cos \left[\left(\Omega+\omega_{3}\right) \tau+\left(\beta_{1}+\beta_{3}\right)\right]\right\} \\
J_{5}= & -\frac{\delta a_{1}^{\prime} \omega_{1}}{2}\left\{\cos \left[\left(\Omega-\omega_{3}\right) \tau+\left(\beta_{1}-\beta_{3}\right)\right]\right. \\
& \left.-\cos \left[\left(\Omega+\omega_{3}\right) \tau+\left(\beta_{1}+\beta_{3}\right)\right]\right\} \\
J_{6}= & -\frac{\delta a_{1} \omega_{1} \beta_{1}^{\prime}}{2}\left\{\sin \left[\left(\Omega+\omega_{3}\right) \tau+\left(\beta_{1}+\beta_{3}\right)\right]\right. \\
& \left.-\sin \left[\left(\Omega-\omega_{3}\right) \tau+\left(\beta_{1}-\beta_{3}\right)\right]\right\}
\end{aligned}
$$




$$
\begin{aligned}
J_{7}= & -\frac{\delta a_{1}}{2}\left(\omega_{1} \Omega-\Omega_{3}^{2}\right)\left\{\sin \left[\left(\Omega+\omega_{3}\right) \tau+\left(\beta_{1}+\beta_{3}\right)\right]\right. \\
& \left.-\sin \left[\left(\Omega-\omega_{3}\right) \tau+\left(\beta_{1}-\beta_{3}\right)\right]\right\}
\end{aligned}
$$

$$
\begin{aligned}
J_{1}^{\prime}= & \frac{a_{1}^{\prime} \omega_{1}}{2 a_{3} \omega_{3}}\left\{\sin \left[\left(\Omega+\omega_{3}\right) \tau+\left(\beta_{1}+\beta_{3}\right)\right]\right. \\
& \left.-\cos \left[\left(\omega_{3}-\Omega\right) \tau+\left(\beta_{3}-\beta_{1}\right)\right]\right\}
\end{aligned}
$$

$$
J_{2}^{\prime}=\frac{a_{1} \beta_{1}^{\prime} \omega_{1}}{2 a_{3} \omega_{3}}\left\{\cos \left[\left(\Omega+\omega_{3}\right) \tau+\left(\beta_{1}+\beta_{3}\right)\right]\right.
$$$$
\left.+\cos \left[\left(\Omega-\omega_{3}\right) \tau+\left(\beta_{1}-\beta_{3}\right)\right]\right\}
$$

$J_{3}^{\prime}=\frac{a_{1} \omega_{1} \Omega}{2 a_{3} \omega_{3}}\left\{\cos \left[\left(\Omega+\omega_{3}\right) \tau+\left(\beta_{1}+\beta_{3}\right)\right]\right.$$$
\left.+\cos \left[\left(\Omega-\omega_{3}\right) \tau+\left(\beta_{1}-\beta_{3}\right)\right]\right\}
$$

$$
J_{4}^{\prime}=-\varepsilon \frac{\mu_{3}}{2} \sin \left[2\left(\omega_{3} \tau+\beta_{3}\right)\right]
$$

$$
\begin{aligned}
J_{5}^{\prime}= & -\varepsilon \frac{\mu_{3} \delta a_{1}^{\prime} \omega_{1}}{2 a_{3} \omega_{3}}\left\{\sin \left[\left(\Omega+\omega_{3}\right) \tau+\left(\beta_{1}+\beta_{3}\right)\right]\right. \\
& \left.-\sin \left[\left(\omega_{3}-\Omega\right) \tau+\left(\beta_{3}-\beta_{1}\right)\right]\right\}
\end{aligned}
$$

$$
\begin{aligned}
J_{6}^{\prime}= & -\frac{\delta a_{1}^{\prime} \omega_{1}}{2 a_{3} \omega_{3}}\left\{\sin \left[\left(\Omega+\omega_{3}\right) \tau+\left(\beta_{1}+\beta_{3}\right)\right]\right. \\
& \left.-\sin \left[\left(\omega_{3}-\Omega\right) \tau+\left(\beta_{1}-\beta_{3}\right)\right]\right\}
\end{aligned}
$$

$$
\begin{aligned}
J_{7}^{\prime}= & -\frac{\delta a_{1} \omega_{1} \beta_{1}^{\prime}}{2 a_{3} \omega_{3}}\left\{\cos \left[\left(\Omega+\omega_{3}\right) \tau+\left(\beta_{1}+\beta_{3}\right)\right]\right. \\
& \left.+\sin \left[\left(\Omega-\omega_{3}\right) \tau+\left(\beta_{1}-\beta_{3}\right)\right]\right\}
\end{aligned}
$$

$$
J_{8}^{\prime}=-\frac{\delta a_{1}}{2 a_{3} \omega_{3}}\left(\omega_{1} \Omega-\omega_{3}^{2}\right)\left\{\cos \left[\left(\Omega+\omega_{3}\right) \tau+\left(\beta_{1}+\beta_{3}\right)\right]\right.
$$$$
\left.+\cos \left[\left(\Omega-\omega_{3}\right) \tau+\left(\beta_{1}-\beta_{3}\right)\right]\right\}
$$

$$
G_{1}=\left(\frac{a_{3}^{2} \omega_{3}^{2}}{2}+\frac{a_{1}^{2} \delta^{2} a_{3}}{4}\right)\left\{\sin \left[\left(2 \Omega+2 \omega_{3}\right) \tau+\left(\beta_{1}+3 \beta_{3}\right)\right]\right.
$$$$
\left.-\sin \left[\left(\omega_{3}-\Omega\right) \tau+\left(\beta_{3}-\beta_{1}\right)\right]\right\}
$$

$$
\begin{aligned}
G_{2}= & \left(-\frac{a_{3}^{2} \omega_{3}^{2}}{2}\right)\left\{\sin \left[\left(\Omega+3 \omega_{3}\right) \tau+\left(\beta_{1}+3 \beta_{3}\right)\right]\right. \\
& \left.-\sin \left[\left(3 \omega_{3}-\Omega\right) \tau+\left(3 \beta_{3}-\beta_{1}\right)\right]\right\}
\end{aligned}
$$

$$
G_{3}=\left(-\frac{a_{3}^{2} \omega_{3} a_{1} \delta}{4}\right)\left\{\sin \left[\left(2 \Omega+2 \omega_{3}\right) \tau+\left(2 \beta_{1}+2 \beta_{3}\right)\right]\right.
$$

$$
\left.-\sin \left(2 \omega_{3} \tau+2 \beta_{3}\right)\right\}
$$

$$
\begin{aligned}
G_{4}= & \left(-\frac{a_{1}^{2} \delta^{3}}{8}\right)\left\{\sin \left[\left(3 \Omega+\omega_{3}\right) \tau+\left(3 \beta_{1}+\beta_{3}\right)\right]\right. \\
& \left.-\sin \left[\left(\Omega+\omega_{3}\right) \tau+\left(\beta_{3}+\beta_{1}\right)\right]\right\}
\end{aligned}
$$

$$
\begin{aligned}
G_{5}= & \left(-\frac{a_{1}^{2} \delta^{2} a_{3}}{8}\right)\left\{\sin \left[\left(3 \Omega-\omega_{3}\right) \tau+\left(3 \beta_{1}-\beta_{3}\right)\right]\right. \\
& \left.-\sin \left[\left(\Omega-\omega_{3}\right) \tau+\left(\beta_{1}-\beta_{3}\right)\right]\right\} \\
G_{6}= & \left(\frac{a_{3}^{2} \omega_{3}^{2} a_{1} \delta}{4}+\frac{a_{3}^{2} \delta^{2}}{2}-\frac{a_{1}^{3} \delta^{3}}{8}\right)\left[\sin \left(2 \Omega \tau+2 \beta_{1}\right)\right]
\end{aligned}
$$

$$
\begin{aligned}
G_{7}= & \left(-\frac{a_{3}^{2} \omega_{3}^{2} \delta a_{1}}{8}\right)\left\{\sin \left[\left(2 \Omega+2 \omega_{3}\right) \tau+\left(2 \beta_{1}+2 \beta_{3}\right)\right]\right. \\
& \left.-\sin \left(2 \omega_{3} \tau+2 \beta_{3}\right)\right\}
\end{aligned}
$$

$$
\begin{aligned}
G_{8}= & \left(-\frac{a_{3}^{2} \omega_{3}^{2} \delta a_{1}}{8}\right)\left\{\sin \left(2 \omega_{3} \tau+2 \beta_{3}\right)\right. \\
& \left.-\sin \left[\left(2 \omega_{3}-2 \Omega\right) \tau+\left(2 \beta_{3}-2 \beta_{1}\right)\right]\right\}
\end{aligned}
$$

$$
\begin{aligned}
G_{9}= & \left(\frac{a_{1}^{2} \delta^{2} a_{3} \omega_{3}}{4}\right)\left\{\sin \left[\left(3 \Omega-\omega_{3}\right) \tau+\left(3 \beta_{1}-\beta_{3}\right)\right]\right. \\
& \left.-\sin \left[\left(\Omega-\omega_{3}\right) \tau+\left(\beta_{1}-\beta_{3}\right)\right]\right\}
\end{aligned}
$$

$$
\begin{aligned}
G_{10}= & \left(-\frac{a_{1}^{2} \delta a_{3} \omega_{3}}{4}\right)\left\{\sin \left[\left(3 \Omega+\omega_{3}\right) \tau+\left(3 \beta_{1}+\beta_{3}\right)\right]\right. \\
& \left.-\sin \left[\left(\Omega+\omega_{3}\right) \tau+\left(\beta_{1}+\beta_{3}\right)\right]\right\}
\end{aligned}
$$

$$
G_{11}=\left(\frac{a_{1} \delta a_{3} \omega_{3}}{8}\right)\left\{\sin \left(4 \Omega \tau+4 \beta_{1}\right)-\sin \left(2 \Omega \tau+2 \beta_{1}\right)\right\}
$$

$$
\begin{aligned}
G_{12}= & \left(\frac{a_{3}^{\prime} \omega_{3}}{2}\right)\left\{\cos \left[\left(\omega_{3}-\Omega+\right) \tau+\left(\beta_{3}-\beta_{1}\right)\right]\right. \\
& \left.-\sin \left[\left(\omega_{3}-\Omega\right) \tau+\left(\beta_{3}-\beta_{1}\right)\right]\right\}
\end{aligned}
$$

$$
\begin{aligned}
G_{13}= & \left(\frac{a_{3} \omega_{3} \beta_{3}^{\prime}}{2}\right)\left\{\sin \left[\left(\omega_{3}+\Omega\right) \tau+\left(\beta_{3}+\beta_{1}\right)\right]\right. \\
& \left.-\sin \left[\left(\omega_{3}-\Omega\right) \tau+\left(\beta_{3}-\beta_{1}\right)\right]\right\}
\end{aligned}
$$




$$
\begin{aligned}
G_{14}= & \left(\frac{a_{3} \omega_{3}^{2}}{2}\right)\left\{\sin \left[\left(\omega_{3}+\Omega\right) \tau+\left(\beta_{3}+\beta_{1}\right)\right]\right. \\
& \left.-\sin \left[\left(\omega_{3}-\Omega\right) \tau+\left(\beta_{3}-\beta_{1}\right)\right]\right\} \\
G_{1}^{\prime}= & \left(\frac{a_{3}^{3} \omega_{3}^{2}}{4}-\frac{a_{3}^{3} \omega_{3}^{3}}{8}+\frac{a_{1}^{2} \delta^{2} \omega_{1}^{2} a_{3}}{4}\right)\left\{\cos \left[\left(\Omega+\omega_{3}\right) \tau+\left(\beta_{1}+\beta_{3}\right)\right]\right\} \\
G_{2}^{\prime}= & \left(-\frac{a_{3}^{3} \omega_{3}^{2}}{4}\right)\left\{\cos \left[\left(3 \omega_{3}+\Omega\right) \tau+\left(3 \beta_{3}+\beta_{1}\right)\right]\right. \\
& \left.+\cos \left[\left(3 \omega_{3}-\Omega\right) \tau+\left(3 \beta_{3}-\beta_{1}\right)\right]\right\}
\end{aligned}
$$

\section{References}

1. Kononenko VO (1969) Vibrating systems with a limited power supply. Iliffe Books Ltd, London

2. Nayfeh AH (1981) Introduction to Perturbation Techniques. John Wiley \& Sons Inc, New York

3. Danuta S, Kot M (2007) Chaotic vibration of an autoparametrical system with a non-ideal source of power. J Theor Appl Mech 45(1):119-131

4. Balthazar JM, Mook DT, Weber HI, Brasil RMLRF, Fenili A, Belato D, Felix JLP (2001) Recent results on vibrating problems with limited power supply. In: Sixth Conference on dynamical Systems Theory and Applications, Lodz, Poland, December, 10-12, 27-50

5. Gonçalves PJP, Silveira M, Pontes Junior BR, Balthazar JM (2014) The dynamic behavior of a cantilever beam coupled to a non-ideal unbalanced motor through numerical and experimental analysis. J Sound Vib 333:5115-5129

$$
\begin{aligned}
& G_{3}^{\prime}=\left(\frac{a_{3}^{2} \omega_{3} a_{1} \delta}{4}\right)\left\{\begin{array}{l}
{\left[\cos \left(2 \omega_{3} \tau\right)+\cos \left(2 \omega_{3}-2 \Omega\right) \tau+\left(2 \beta_{3}-2 \beta_{1}\right)\right]} \\
-\left[\cos \left[\left(2 \omega_{3}+2 \Omega\right) \tau+\left(3 \beta_{3}+2 \beta_{1}\right)\right]+\cos \left(2 \omega_{3} \tau+2 \beta_{3}\right)\right]
\end{array}\right\} \\
& G_{4}^{\prime}=\left(-\frac{a_{1}^{2} \omega_{1}^{3} a_{3} \delta^{2}}{8}\right)\left\{\begin{array}{l}
{\left[\cos \left(\left(3 \Omega+\omega_{3}\right) \tau+\left(3 \beta_{1}+\beta_{3}\right)\right)+\cos \left(\left(\Omega+\omega_{3}\right) \tau\right)\right]} \\
+\left[\cos \left[\left(2 \Omega-\omega_{3}\right) \tau+\left(3 \beta_{1}-\beta_{3}\right)\right]+\cos \left[\left(\Omega-\omega_{3}\right) \tau+\left(\beta_{1}-\beta_{3}\right)\right]\right]
\end{array}\right\}
\end{aligned}
$$

$$
\begin{aligned}
& G_{5}^{\prime}=\left(\frac{a_{3}^{2} \omega_{3} a_{1} \delta}{4}+\frac{a_{3}^{3} \omega_{1}^{2} \delta^{3}}{4}-\frac{a_{1}^{3} \delta^{3} \omega_{1}^{2}}{8}\right)\left[1-\cos \left(2 \Omega \tau+2 \beta_{1}\right)\right] \\
& G_{6}^{\prime}=\left(-\frac{a_{3}^{2} \omega_{3}^{2} a_{1} \delta}{8}\right)\left\{\begin{array}{l}
{\left[\cos \left(\left(2 \Omega+\omega_{3}\right) \tau+\left(2 \beta_{1}+2 \beta_{3}\right)\right)+\cos \left(2 \omega_{3} \tau+2 \beta_{3}\right)\right]} \\
+\left[\cos \left(2 \omega_{3} \tau+2 \beta_{3}\right)+\cos \left[\left(2 \omega_{3}-2 \Omega\right) \tau+\left(2 \beta_{3}-2 \beta_{1}\right)\right]\right]
\end{array}\right\} \\
& G_{7}^{\prime}=\left(\frac{a_{1}^{2} \omega_{3}^{2} a_{3} \delta}{4}\right)\left\{\begin{array}{l}
{\left[\cos \left(\left(3 \Omega-\omega_{3}\right) \tau+\left(3 \beta_{1}-\beta_{3}\right)\right)+\cos \left(\left(\Omega-\omega_{3}\right) \tau+\left(\beta_{1}-\beta_{3}\right)\right)\right]} \\
-\left[\cos \left(\left(\omega_{3}+3 \Omega\right) \tau+\left(3 \beta_{1}+\beta_{3}\right)\right)+\cos \left[\left(\omega_{3}+\Omega\right) \tau+\left(\beta_{3}+\beta_{1}\right)\right]\right]
\end{array}\right\}
\end{aligned}
$$

$$
\begin{aligned}
G_{8}^{\prime}= & \left(-\frac{a_{3}^{3} \omega_{1}^{2} \delta^{3}}{8}\right)\left[\cos \left(4 \Omega \tau+4 \beta_{1}\right)+\cos \left(2 \Omega \tau+2 \beta_{1}\right)\right] \\
& +\left(\frac{a_{3}^{\prime} \omega_{3}}{2}\right)\left[\sin \left(\left(\Omega+\omega_{3}\right) \tau+\left(\beta_{1}+\beta_{3}\right)\right)\right. \\
& \left.-\sin \left(\left(\Omega-\omega_{3}\right) \tau+\left(\beta_{1}-\beta_{3}\right)\right)\right] \\
G_{9}^{\prime}= & \left(\frac{a_{3} \omega_{3} \beta_{3}^{\prime}}{2}\right)\left\{\cos \left[\left(\Omega+\omega_{3}\right) \tau+\left(\beta_{3}+\beta_{1}\right)\right]\right. \\
& \left.+\cos \left[\left(\omega_{3}-\Omega\right) \tau+\left(\beta_{3}-\beta_{1}\right)\right]\right\} \\
G_{10}^{\prime}= & \left(\frac{a_{3} \omega_{3}^{2}}{2}\right)\left\{\cos \left[\left(\Omega+\omega_{3}\right) \tau+\left(\beta_{3}+\beta_{1}\right)\right]\right. \\
& \left.+\cos \left[\left(\omega_{3}-\Omega\right) \tau+\left(\beta_{3}-\beta_{1}\right)\right]\right\}
\end{aligned}
$$

6. Cveticanin L, Zukovic M (2015) Motion of a motor-structure non-ideal system. Eur J Mech A Solids 53:229-240

7. Fossi DOT, Woafo P (2013) Generation of complex phenomena in a simple electromechanical system using the feedback control. Commun Nonlinear Sci Numer Simulat 18:209-218

8. Palacios JL, Balthazar JM, Brasil RMLRF (2002) On nonideal and nonlinear portal frame dynamics analysis using bogoliubov averaging method. J Braz Soc Mech Sci Eng 24(4):257-265

9. Felix JLP, Balthazar JM, Brasil RMLRF (2005) On saturation control of a non-ideal vibrating portal frame foundation type shear-building. J Vib Control 11:121-136

10. Arbex HC, Balthazar JM, Pontes BR Jr, Brasil RMLRF, Felix JLP, Tusset AM, Bueno AM (2015) On nonlinear dynamics behavior and control of a new model of a magnetically levitated vibrating system, excited by an unbalanced DC motor of limited power supply. J Braz Soc Mech Sci Eng 37(4):1139-1150

11. Piccirillo V, Tusset AM, Balthazar JM (2014) Dynamical jump attenuation in a non-ideal system through magneto rheological damper. J Theor Appl Mech (Warsaw) 53:595-604 
12. Djanan AAN, Nbendjo BRN, Woafo P (2013) Electromechanical control of vibration on a plate submitted to anon-ideal excitation. Mech Res Commun 54:72-82

13. Tusset AM, Balthazar JM, Felix JLP (2013) On elimination of chaotic behavior in a non-ideal portal frame structural system, using both passive and active controls. J Vib Control 19(6):803-813

14. Pearson JD (1962) Approximation methods in optimal control. J Electron Control 13:453-469

15. Wernli A, Cook G (1975) Suboptimal control for the nonlinear quadratic regulator problem. Automatica 11:75-84

16. Mracek CP, Cloutier JR (1998) Control designs for the nonlinear benchmark problem via the state-dependent Riccati equation method. Int J Robust Nonlinear Control 8:401-433

17. Friedland B (1996) Advanced control system design. PrenticeHall, Englewood Cliff, pp 110-112

18. Balthazar JM, Bassinello DG, Tusset AM, Bueno AM, Pontes BR (2014) Nonlinear control in an electromechanical transducer with chaotic behavior. Meccanica 49(8):1859-1867

19. Tusset AM, Balthazar JM (2013) On the chaotic suppression of both ideal and non-ideal duffing based vibrating systems, using a magnetorheological damper. Differ Equ Dyn Syst 21:105-121

20. Bueno AM, Tusset AM, Santos JPM, Ttsushida M, Balthazar JM (2013) SDRE control applied to an electromechanical pendulum excited by a non-ideal motor. In: Proceedings of the ASME 2013 International Design Engineering Technical Conferences \& Computers and Information in Engineering Conference IDETC/ CIE 2013, August 4-7, Portland, Oregon, USA, 2013

21. Tusset MA, Piccirillo V, Bueno AM, Balthazar MJ, Sado D, Felix JLP, Brasil RMLRDF (2015) Chaos control and sensitivity analysis of a double pendulum arm excited by an RLC circuit based nonlinear shaker. J Vib Control 1:1-17

22. Molter A, Silveira OAA, Fonseca JSO, Bottega V (2010) Simultaneous piezoelectric actuator and sensor placement optimization and control design of manipulators with flexible links using SDRE method. Math Problems Eng 2010:362437
23. Fenili A, Balthazar JM (2011) The rigid-flexible nonlinear robotic manipulator: modeling and control. Commun Nonlinear Sci Numer Simul 16(5):2332-2341

24. Lima JJ, Tusset AM, Janzen FC, Piccirillo V, Nascimento CB, Balthazar JM, Brasil MRLF (2014) Nonlinear state estimation and control applied to a manipulator robotic including drive motor. Math Eng Sci Aerosp Transdiscipl Int J 5:413-425

25. Tusset AM, Balthazar JM, Bassinello DG, Pontes BR, Felix JLP (2012) Statements on chaos control designs, including a fractional order dynamical system, applied to a MEMS comb-drive actuator. Nonlinear Dyn 69:1837-1857

26. Tusset AM, Bueno AM, Nascimento CB, Kaster MS, Balthazar JM (2013) Nonlinear state estimation and control for chaos suppression in MEMS resonator. Shock Vib 20:749-761

27. Balthazar JM, Tusset AM, Souza SLTD, Bueno AM (2012) Microcantilever chaotic motion suppression in tapping mode atomic force microscope. Proc Inst Mech Eng Part C J Mech Eng Sci 227:1730-1741

28. Nozaki R, Balthazar JM, Tusset AM, Pontes BR, Bueno AM (2013) Nonlinear control system applied to atomic force microscope including parametric errors. J Control Autom Electri Syst 24:223-231

29. Rodrigues KS, Balthazar JM, Tusset AM, Pontes BR, Bueno AM (2014) Preventing chaotic motion in tapping-mode atomic force microscope. J Control Autom Electri Syst 25:732-740

30. Balthazar JM, Tusset AM, Bueno AM (2014) Nonlinear TMAFM control considering parametric errors in the control signal evaluation. J Theor Appl Mech (Warsaw) 52:93-106

31. Felix JLP, Balthazar JM, Brasil RMLRF (2009) Comments on nonlinear dynamics of a non-ideal Duffing-Rayleigh oscillator: numerical and analytical approachs. J Sound Vib 319:1136-1149

32. El-Badawy AA (2007) Behavioral investigation of a nonlinear vibrating system. J Vib Control 13(2):203-217

33. Kuo BC (1995) Automatic control systems. Prentice Hall, New Jersey 